\title{
Pre-treatment inflammatory biomarkers predict
} early treatment response and favorable survival in patients with metastatic colorectal cancer who underwent first line cetuximab plus chemotherapy

This article was published in the following Dove Press journal:

Cancer Management and Research

\author{
Jinling Jiang' \\ Tao Ma' \\ Wenqi $\mathrm{Xi}^{\mathrm{I}}$ \\ Chen Yang' \\ Junwei $\mathrm{Wu}^{\prime}$ \\ Chenfei Zhou' \\ Nan Wang' \\ Zhenggang Zhu' ${ }^{1,2}$ \\ Jun Zhang ${ }^{1,2}$ \\ 'Department of Oncology, Ruijin \\ Hospital, Shanghai Jiao Tong University \\ School of Medicine, Shanghai 200025, \\ People's Republic of China; ${ }^{2}$ Shanghai \\ Institute of Digestive Surgery, Ruijin \\ Hospital, Shanghai Jiao Tong University \\ School of Medicine, Shanghai 200025, \\ People's Republic of China
}

Correspondence: Jun Zhang Department of Oncology, Ruijin Hospital, Shanghai Jiao Tong University School of Medicine, Shanghai 200025, People's

Republic of China

$\mathrm{Tel} / \mathrm{Fax}+862164741635$

Email junzhang10977@sjtu.edu.cn
Objective: This study was to determine whether peripheral blood biomarkers including neutrophil-lymphocyte ratio (NLR), platelet-lymphocyte ratio (PLR), and systemic immune inflammation index (SII) could predict early response to cetuximab; moreover, the prognostic ability of those biomarkers on progression free survival (PFS) and overall survival (OS) of metastatic colorectal cancer (mCRC) patients with wild-type (WT) RAS was also investigated. Methods: mCRC patients with WT RAS treated with cetuximab plus chemotherapy were retrospectively analyzed, and early response was evaluated according to RECIST 1.1 after three or four treatment cycles. In prior to chemotherapy, hematologic data and clinic-pathological parameters were collected. The associations between pre-treatment inflammatory biomarkers and early response, and the prognostic value of those biomarkers were analyzed. A total of 102 patients were enrolled and divided into low or high NLR, PLR, and SII groups, respectively.

Results: The early response rate was significantly higher in the low NLR $(p<0.001)$, low PLR ( $p=0.045$ ), and low SII ( $p=0.011)$, respectively. In multivariate analyses, primary tumor resection (hazard ratio (HR) $0.411, p<0.001$ ), carcino-embryonicantigen $\leq 5 \mathrm{ng} / \mathrm{mL}$ (HR $0.406, p<0.001$ ), early treatment response (HR 0.322, $p<0.001$ ), and low NLR (HR 0.665, $p=0.031$ ) were independent factors of longer PFS. Primary tumor resection (HR 0.488 , $p=0.003$ ) and early response (HR $0.392, p<0.001$ ) were independent factors of longer OS. Further analysis showed that patients with early response, even in the high groups, can achieve better PFS and OS than non-responders.

Conclusion: Pre-treatment inflammatory biomarkers, especially NLR were predictors of benefit from cetuximab-combined therapy in mCRC patients. They were also predictors of significantly longer PFS and OS of early responders compared to non-responders.

Keywords: inflammatory biomarkers, cetuximab, early treatment response, wild-type RAS, metastatic colorectal cancer

\section{Introduction}

Recently, the prognosis of locally advanced or metastatic colorectal cancer (mCRC) with wild-type (WT) RAS had dramatically improved due to the introduction of cetuximab. ${ }^{1-4}$ Cetuximab is a monoclonal antibody which targeting the transmembrane protein epidermal growth factor receptor (EGFR), leading to the inhibition of the MAPK pathway and therefore suppresses tumor cell differentiation, proliferation, and angiogenesis which 
contributes to tumor progression..$^{5}$ Although several mechanisms of primary or acquired resistance had been identified, the only established response predictive biomarker for the treatment of mCRC patients is the RAS mutational status. ${ }^{6}$ Moreover, even RAS WT patients who initially responded to anti-EGFR therapy eventually would undergo tumor progression, suggesting unknown alternative mechanisms capable of influencing treatment effectiveness were still existed. In this regard, identifying more sensitive markers for predicting therapeutic efficacy to promote the development of individualized treatment is urgently needed.

It has been increasingly recognized that tumor growth and metastasis resulted from interactions between tumoral and stromal factors, including blood vessels, inflammatory cells, and immunity system which led to an inflammation status. ${ }^{7,8}$ Markers such as C-reactive protein, hypoalbuminemia, Glasgow Prognostic Score, neutrophil count (PNC), macrophage, neutrophil-lymphocyte ratio (NLR), platelet-lymphocyte ratio (PLR), and systemic immune-inflammation index (SII), have been investigated as prognostic and predictive factors in various human cancer types, especially in radically resected or mCRC. ${ }^{9-14}$ There is increasing evidence that inflammation markers served as an important role in the induction of chemo-resistance. ${ }^{15-18}$ Also, certain inflammatory indexes were correlated with chemotherapeutic responses. Van Glabbeke et al, ${ }^{19}$ demonstrated that an elevated baseline neutrophil count correlated with initial and late resistance to imatinib treatment in gastrointestinal stromal tumors. High PNC and NLR values were associated with chemo-resistance and an unfavorable prognosis in patients with stage III and IV unresectable lung cancer. ${ }^{20}$ Elevated baseline NLR correlated with poor response treated with bevacizumab plus chemotherapy in mCRC. ${ }^{21}$ Recently, elevated pre-treatment NLR could serve as a predictor of survival and cetuximab efficacy in mCRC patients with WT RAS. ${ }^{22}$ However, the relationship between inflammatory biomarker, early treatment response, and cetuximab efficacy is still yet to be known.

In this single-center, retrospective study, we aimed to investigate pre-treatment parameters including NLR, PLR, and SII for their ability to predict early treatment response and survival of mCRC patients receiving first-line chemotherapy plus cetuximab.

\section{Materials and methods}

\section{Patient and data collection}

We retrospectively enrolled 102 patients who were diagnosed with primary colorectal cancer and received chemotherapy plus cetuximab as the initial treatment at Shanghai Jiaotong University School of Medicine Affiliated Ruijin Hospital between January 2010 and December 2017. This study was approved by the Medical Ethical Committee of Shanghai Ruijin Hospital and performed in accordance with relevant guidelines and regulations. Patients alive signed an informed consent for the use of their personal data for research purposes at the time of data collection. For patients who have died at the time of data collection, we had followed up the recurrence or death time by telephone, informed the related contents of the informed consent form of this study in detail, obtained the consent of patients' relatives, and archived the telephone recording, which was approved by the Medical Ethics Committee of our hospital.

The primary inclusion criteria were as follows: (a) histologically confirmed and measurable (RECIST criteria v.1.1) unresectable metastatic adenocarcinoma of the colon or rectum, (b) molecular test showing no mutation in the RAS gene of colorectal carcinoma cells, (c) patients with available and complete basic characteristics, laboratory data, and follow-up information. Patients with evidence for one mutation of the RAS gene, prior chemotherapy for metastatic disease, previous exposure to EGFR-targeting therapy, hematology, and infection diseases were excluded. Patients' demographic and clinic-pathological variables, including age, sex, tumor localization, primary tumor status, adjuvant therapy, tumor metastasis period, liver metastases, carcino-embryonicantigen (CEA), carbohydrate antigen 19-9 (CA199), lactate dehydrogenase (LDH), chemotherapy regimen, and early treatment response were collected using electronic medical records. Laboratory data were obtained within 3 days prior to the initial administration of cetuximab. Blood cell counting was detected by Sysmex hematology analyzers. NLR and PLR were defined as the absolute counts of neutrophils and platelets, respectively, divided by the absolute lymphocyte count. SII was calculated as platelet count $\times$ neutrophil count/lymphocyte count. ${ }^{22}$

\section{Response assessment}

Response was assessed every three or four treatment cycles using the revised Response Evaluation Criteria in Solid Tumors (version 1.1). ${ }^{23}$ The criteria classified the responses into four categories: Complete response (CR), partial response $(\mathrm{PR})$, stable disease $(\mathrm{SD})$, and progressive disease (PD). CR was defined as disappearance of all target lesions, any pathological lymph nodes (whether target or non-target) must have reduction in short axis to $<10 \mathrm{~mm}$. PR was 
defined as at least a $30 \%$ decrease in the sum of diameters of target lesions, taking as reference the baseline sum diameters. SD was defined as neither sufficient shrinkage to qualify for PR nor sufficient increase to qualify for SD, taking as reference the smallest sum diameters while on study. PD was defined as at least a $20 \%$ increase in the sum of diameters of target lesions, taking as reference the smallest sum on study (this includes the baseline sum if that is the smallest on study). In addition to the relative increase of $20 \%$, the sum must also demonstrate an absolute increase of at least $5 \mathrm{~mm}$. (Note: the appearance of one or more new lesions is also considered progression). Early treatment response was defined as the results of the first efficacy evaluation included CR and PR, and non-response included $\mathrm{SD}$ and PD. The early response rate was the ratio of early response patients to the total patients.

\section{Statistical analysis}

Progression-freesurvival (PFS) was measured as the time between treatment initiation and disease progression or death from any cause. Overall survival (OS) was defined as the time between treatment initiation and death from any cause or the date of last follow-up.

The optimal cut-off values for NLR, PLR, and SII were performed according to the early response by receiver operating characteristic curves, which were used to detect the value of each index for predicting the response to therapy. Patients' characteristics were analyzed by descriptive statistics. The $\chi^{2}$ or Fisher's exact test were used to assess the association between categorical variables. PFS and OS were calculated according to the Kaplan-Meier method, and the log-rank test was used to compare survival between different patient populations. The impact of prognostic factors on PFS and OS was first assessed in univariate analysis by means of the Cox proportional hazard regression analysis, variables with statistically significant in univariate analysis were further analyzed in multivariate analysis. Hazard ratios (HRs) estimated from the Cox proportional hazard model were reported as relative risks with corresponding 95\% Confidence Intervals (95\% CI). All statistical analyses were performed using the SPSS version 20.0 (IBM Corporation, Armonk, NY, USA). $P<0.05$ was considered to indicate a statistically significant difference.

\section{Results}

\section{Patient characteristics}

Among 102 patients treated with cetuximab, patients were divided into high and low index groups on the basis of the specified cut-off value of NLR (3.285, AUC $=0.701)$, PLR (171.45, AUC $=0.569)$, and SII (660.55, AUC $=0.619)$, respectively (Figure 1). NLR $\geq 3.285$, PLR $\geq 171.45$, and SII $\geq 660.55$ were considered as high groups.

All the clinic-pathological characteristics of patients are detailed in Table 1. There are 72 (70.6\%) males and $30(29.4 \%)$ females, with $45(44.1 \%)$ patients were $>60$ years and 57 patients were $\leq 60$ (55.9\%; range, 28-75 years). According to the location of the tumor, most of them $(80,78.5 \%)$ occurred in the left colon while other 22 $(21.5 \%)$ were from right colon. Primary tumor resection was performed in $76(74.5 \%)$ of all patients and 23 $(37.3 \%)$ of them underwent adjuvant chemotherapy. Sixty-five $(63.7 \%)$ patients suffered simultaneous metastasis while of which 37 (36.3\%) were metachronism. Among the 102 patients, liver metastasis occurred in 38 $(37.3 \%)$ and other $64(62.7 \%)$ without liver metastasis. Further, $41(40.2 \%)$ patients were with elevated CEA; 45 (44.1\%) patients were with elevated CA199, respectively; $45(44.1 \%)$ patients were with increased LDH, respectively. All the 102 patients received chemotherapy, of which 43 (42.1\%) treated with FOLFOX/XELOX and 59 $(57.9 \%)$ treated with FOLFIRI. Regarding early treatment response, no patients achieved CR, 53 patients achieved $\mathrm{PR}, 33$ patients were $\mathrm{SD}$, and 16 patients were $\mathrm{PD}$. In total 102 patients, $53(52.0 \%)$ patients were defined as responder and $49(48.0 \%)$ patients as non-responder.

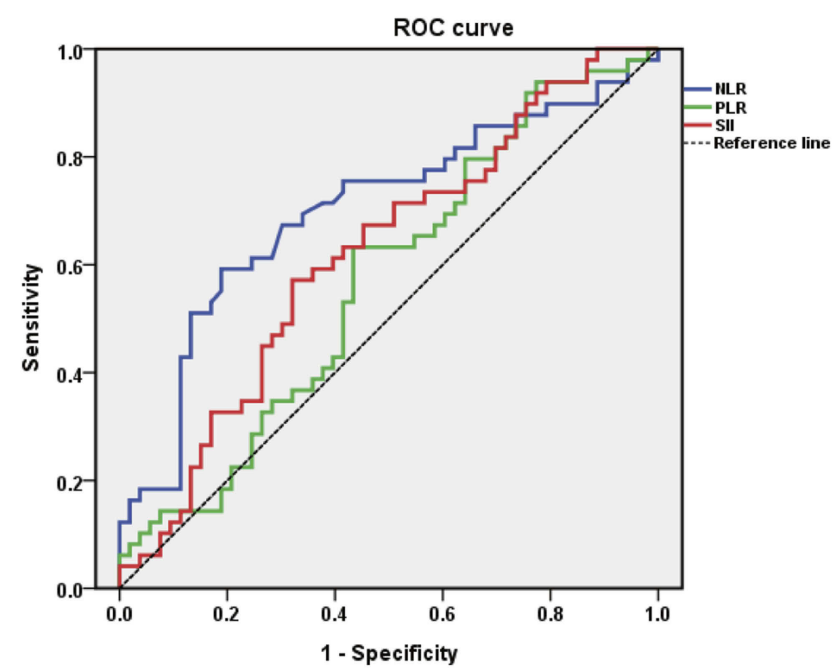

Figure I Diagnostic value of inflammatory biomarkers for early response according to ROC curves.

Abbreviations: ROC, receiver operating characteristic; NLR, neutrophil-to-lymphocyte ratio; PLR, platelet-to-lymphocyte ratio; SII, systemic immune-inflammation index. 
Table I Association between inflammatory markers and clinic-pathological data

\begin{tabular}{|c|c|c|c|c|c|c|c|c|c|c|}
\hline \multirow[t]{2}{*}{ Characteristics } & \multirow{2}{*}{$\begin{array}{l}\text { Total patients } \\
(\mathrm{N}=102)\end{array}$} & \multicolumn{2}{|l|}{ NLR } & \multirow[t]{2}{*}{$P$-value } & \multicolumn{2}{|l|}{ PLR } & \multirow[t]{2}{*}{ P-value } & \multicolumn{2}{|l|}{ sII } & \multirow[t]{2}{*}{$P$-value } \\
\hline & & $\begin{array}{l}\text { Low } \\
(N=63)\end{array}$ & $\begin{array}{l}\text { High } \\
(N=39)\end{array}$ & & $\begin{array}{l}\text { Low } \\
(N=48)\end{array}$ & $\begin{array}{l}\text { High } \\
(N=54)\end{array}$ & & $\begin{array}{l}\text { Low } \\
(N=57)\end{array}$ & $\begin{array}{l}\text { High } \\
(N=45)\end{array}$ & \\
\hline \multicolumn{11}{|l|}{ Age, years } \\
\hline$\leq 60$ & $57(55.9)$ & $36(35.3)$ & $21(20.6)$ & 0.745 & $27(26.5)$ & $30(29.4)$ & 0.944 & $28(27.5)$ & $29(28.4)$ & 0.122 \\
\hline$>60$ & $45(44.1)$ & $27(26.5)$ & $18(17.6)$ & & $21(20.6)$ & $24(23.5)$ & & $29(28.4)$ & $16(15.7)$ & \\
\hline \multicolumn{11}{|l|}{ Sex } \\
\hline Male & $72(70.6)$ & $39(38.2)$ & $33(32.4)$ & 0.014 & $34(33.3)$ & $38(37.3)$ & 0.959 & $40(39.3)$ & $32(31.3)$ & 0.918 \\
\hline Female & $30(29.4)$ & $24(23.6)$ & $6(5.8)$ & & $14(13.7)$ & $16(15.7)$ & & $17(16.7)$ & $13(12.7)$ & \\
\hline \multicolumn{11}{|c|}{ Site of primary tumor (\%) } \\
\hline Right & $22(21.5)$ & $16(15.7)$ & $6(5.8)$ & 0.323 & $9(8.8)$ & $13(12.7)$ & 0.514 & $14(13.7)$ & $8(7.8)$ & 0.408 \\
\hline Left & $80(78.5)$ & $47(46.1)$ & $33(32.4)$ & & $39(38.2)$ & $41(40.3)$ & & $43(42.2)$ & $37(36.3)$ & \\
\hline \multicolumn{11}{|c|}{ Resected primary tumor (\%) } \\
\hline Yes & $76(74.5)$ & $50(49.0)$ & $26(25.5)$ & 0.153 & $40(39.2)$ & $36(35.3)$ & 0.054 & $46(45.1)$ & $30(29.4)$ & 0.106 \\
\hline No & $26(25.5)$ & $13(12.7)$ & $13(12.7)$ & & $8(7.8)$ & $18(17.7)$ & & II (10.8) & $15(14.7)$ & \\
\hline \multicolumn{11}{|c|}{ Previous adjuvant therapy (\%) } \\
\hline Yes & $38(37.3)$ & $25(24.6)$ & $13(12.7)$ & 0.519 & $15(14.7)$ & $23(22.6)$ & 0.237 & $28(27.5)$ & $10(9.8)$ & 0.005 \\
\hline No & $64(62.7)$ & $38(37.2)$ & $26(25.5)$ & & $33(32.4)$ & $31(30.3)$ & & $29(28.4)$ & $35(34.3)$ & \\
\hline \multicolumn{11}{|c|}{ Time to metastases (\%) } \\
\hline Synchronous & $65(63.7)$ & $4 \mid(40.2)$ & $24(23.5)$ & 0.718 & $30(29.4)$ & $35(34.3)$ & 0.808 & $32(31.3)$ & $33(32.4)$ & 0.073 \\
\hline Metachronous & $37(36.3)$ & $22(21.6)$ & $15(14.7)$ & & $18(17.7)$ & $19(18.6)$ & & $25(24.5)$ & $12(11.8)$ & \\
\hline \multicolumn{11}{|c|}{ Liver metastases (\%) } \\
\hline Yes & $38(37.3)$ & $25(24.6)$ & $13(12.7)$ & 0.519 & $21(20.6)$ & $17(16.7)$ & 0.201 & $24(23.6)$ & $14(13.7)$ & 0.254 \\
\hline No & $64(62.7)$ & $38(37.2)$ & $26(25.5)$ & & $27(26.4)$ & $37(36.3)$ & & $33(32.4)$ & $31(30.3)$ & \\
\hline \multicolumn{11}{|l|}{ CEA (ng/mL) } \\
\hline$\leq 5$ & 17 (16.7) & $10(9.8)$ & $7(6.9)$ & 0.785 & $6(5.9)$ & II (10.8) & 0.287 & II (10.8) & $6(5.9)$ & 0.422 \\
\hline$>5$ & $85(83.3)$ & $53(52.0)$ & $32(31.3)$ & & $42(4 I .2)$ & $43(42.1)$ & & $46(45.1)$ & 39 (38.2) & \\
\hline \multicolumn{11}{|l|}{ CAI $99(\mathrm{U} / \mathrm{mL})$} \\
\hline$\leq 37$ & $4 \mid(40.2)$ & $29(28.4)$ & $12(11.8)$ & 0.127 & $22(21.6)$ & $19(18.6)$ & 0.274 & $27(26.5)$ & 14 (13.7) & 0.096 \\
\hline$>37$ & 61 (59.8) & $34(33.3)$ & $27(26.5)$ & & 26 & $35(34.3)$ & & $30(29.4)$ & $31(30.4)$ & \\
\hline \multicolumn{11}{|l|}{ LDH (IU/mL) } \\
\hline$\leq 192$ & $45(44.1)$ & $32(31.3)$ & $13(12.7)$ & 0.084 & $23(22.5)$ & $22(21.6)$ & 0.466 & $30(29.4)$ & $15(14.7)$ & 0.051 \\
\hline$>192$ & $57(55.9)$ & $31(30.3)$ & $26(25.5)$ & & $25(24.6)$ & $32(31.3)$ & & $27(26.5)$ & $30(29.4)$ & \\
\hline \multicolumn{11}{|l|}{$\mathrm{CT}$ regimen (\%) } \\
\hline $\begin{array}{l}\text { FOLFOXI } \\
\text { XELOX }\end{array}$ & $43(42.1)$ & $30(29.4)$ & $13(12.7)$ & 0.156 & $20(16.6)$ & $24(23.5)$ & 0.777 & $28(27.5)$ & $15(14.7)$ & 0.109 \\
\hline FOLFIRI & $59(57.9)$ & $33(32.4)$ & $26(25.5)$ & & $28(27.5)$ & $30(29.4)$ & & $29(28.4)$ & $30(29.4)$ & \\
\hline \multicolumn{11}{|c|}{ Early treatment response (\%) } \\
\hline Response & $53(52.0)$ & $43(42.2)$ & $10(9.8)$ & $<0.001$ & $30(29.4)$ & $23(22.6)$ & 0.045 & $36(35.3)$ & $17(16.7)$ & 0.011 \\
\hline Non-response & $49(48.0)$ & $20(19.6)$ & $29(28.4)$ & & $18(17.6)$ & 31 (30.4) & & $21(20.5)$ & $28(27.5)$ & \\
\hline
\end{tabular}

Notes: Values in the table are presented as the number of patients with the percentage in parenthesis, unless indicated otherwise, $P$-value $<0.05$ was considered statistically significant. Abbreviations: NLR, neutrophil-to-lymphocyte ratio; PLR, platelet-to-lymphocyte ratio; SIl, systemic immune-inflammation index; CEA, carcino-embryonic antigen; CA199, carbohydrate antigen 19-9; LDH, lactate dehydrogenase; CT, chemotherapy. 


\section{Associations between inflammatory biomarkers and baseline characteristics}

To investigate the correlation of NLR, PLR, and SII with clinic-pathologic parameters, we found that high levels of NLR were significantly associated with fewer females $(p=0.014)$ and more non-responders $(p<0.001)$. Only PLR was significantly associated with early treatment response $(p=0.045)$ while SII was significantly associated with adjuvant therapy $(p=0.005)$ and early treatment response $(p=0.011)$. Conversely, there was no significant association between inflammatory biomarkers and other clinical parameters (Table 1; Figure 2).

\section{Survival analyses}

At a median follow-up of 33.2 months (range: 2.6-94.5), 93 (91.2\%) patients progressed and of which 70 (68.6\%) died. Median PFS was 13.2 months in patients with $\mathrm{NLR}^{\mathrm{Low}}$ group and 7.9 months in those with $\mathrm{NLR}^{\text {High }}$ group (HR $0.53,95 \% \mathrm{CI}$ $0.31-0.91 ; p=0.0206)$. Regarding the SII, PFS prolonged with SII $<660.55$ as compared to SII $\geq 660.55(11.8 \mathrm{~ms}$ vs $9.5 \mathrm{~ms}$, HR: $0.60,95 \%$ CI: $0.37-0.98, p=0.0424)$. However, there was no significant difference in PFS between PLR ${ }^{\text {High }}$ and PLR ${ }^{\text {Low }}$ groups (HR: 0.85, 95\% CI: 0.53-1.37; $p=0.5137$ ) (Figure 3).

In univariate analysis, factors associated with PFS were: right colon cancer, unresectable primary tumor, CEA $>5 \mathrm{ng} /$ $\mathrm{mL}$, early treatment with non-response, $\mathrm{NLR}^{\mathrm{High}}$, and $\mathrm{SII}^{\mathrm{High}}$ (Figure 4A). Further, those factors were integrated into multivariate analysis which showed that primary tumor resection (HR: 0.411, 95\% CI: 0.264-0.641), CEA $\leq 5 \mathrm{ng} / \mathrm{mL}$ (HR: $0.406,95 \%$ CI: $0.263-0.625$ ), early treatment response (HR: $0.322,95 \%$ CI: $0.218-0.472$ ) and NLR $^{\text {Low }}$ (HR: $0.665,95 \%$ CI: 0.447-0.902) were independent predictors of PFS while other covariates were of no statistically significant (Figure 4B).

Median OS in NLR ${ }^{\text {Low }}$ group was significantly longer than $\mathrm{NLR}^{\text {High }}$ group $(28.3 \mathrm{~m}$ vs $18.3 \mathrm{~m}$, HR: $0.48,95 \% \mathrm{CI}$ : $0.28-0.83, p=0.0083)$. In contrast, there was no statistical significance difference in OS between other groups $\left(\mathrm{PLR}^{\mathrm{High}}\right.$ vs PLR ${ }^{\mathrm{Low}}$, HR: 0.94 and $\mathrm{SII}^{\mathrm{High}}$ and $\mathrm{SII}^{\mathrm{Low}}$, HR: 0.68 , respectively) (Figure 5). In univariate analysis, age ( $p=0.033)$, primary tumor resection $(p=0.041)$, liver metastases $(p=0.036)$, chemotherapy regimen $(p=0.048)$, early treatment response $(p<0.001)$, NLR $(p=0.011)$, and SII
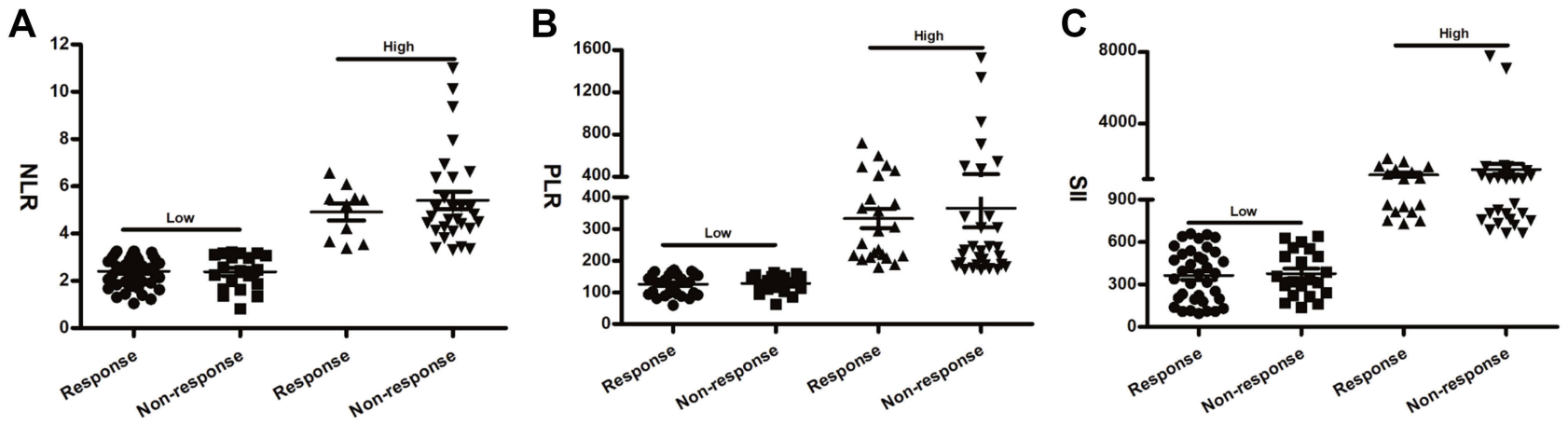

Figure 2 Distribution of NLR (A), PLR (B), and SII (C) according to early response.

Abbreviations: NLR, neutrophil-to-lymphocyte ratio; PLR, platelet-to-lymphocyte ratio; SII, systemic immune-inflammation index.

A

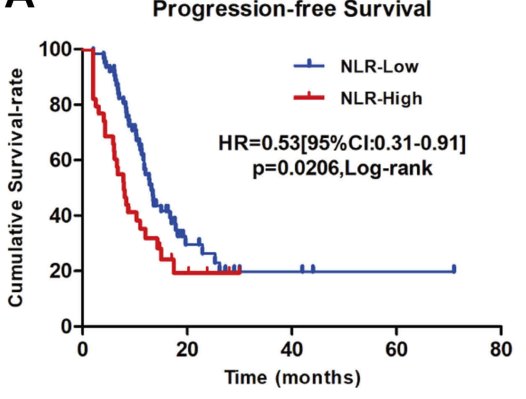

B

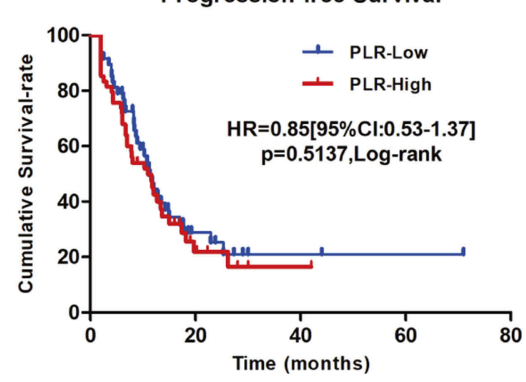

C

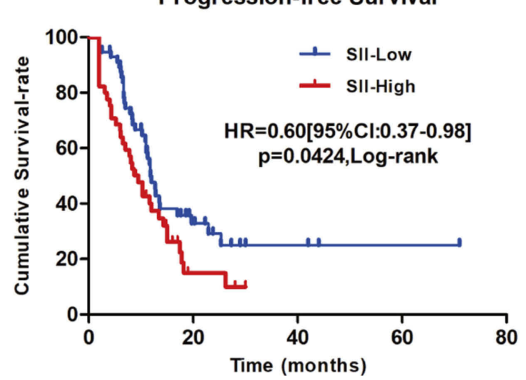

Figure 3 Kaplan-Meier curves of progression-free survival (PFS) of mCRC patients according to baseline NLR (A), PLR (B), and SII (C).

Abbreviations: NLR, neutrophil-to-lymphocyte ratio; PLR, platelet-to-lymphocyte ratio; SIl, systemic immune-inflammation index; mCRC, metastatic colorectal cancer. 

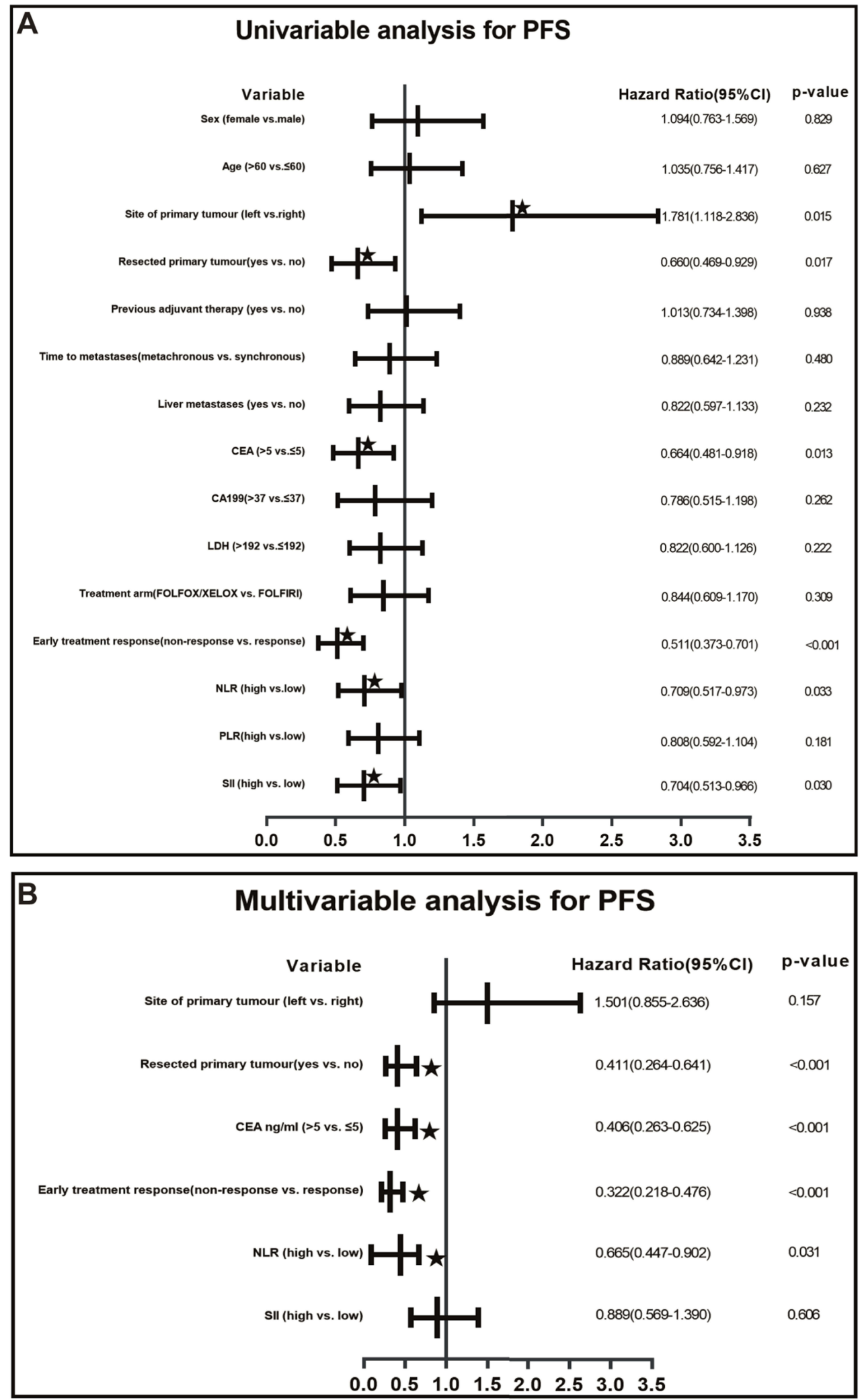

Figure 4 Forest plot illustrating the results of univariable $(\mathbf{A})$ and multivariable $(\mathbf{B})$ analysis of covariates associated with the risk of disease progression in $\mathrm{mCRC}$. $\star$ means $P<0.05$. Abbreviations: PFS, progression free survival; CEA, carcino-embryonic antigen; CAI99, carbohydrate antigen 19-9; LDH, lactate dehydrogenase; NLR, neutrophil-tolymphocyte ratio; PLR, platelet-to-lymphocyte ratio; SII, systemic immune-inflammation index. 

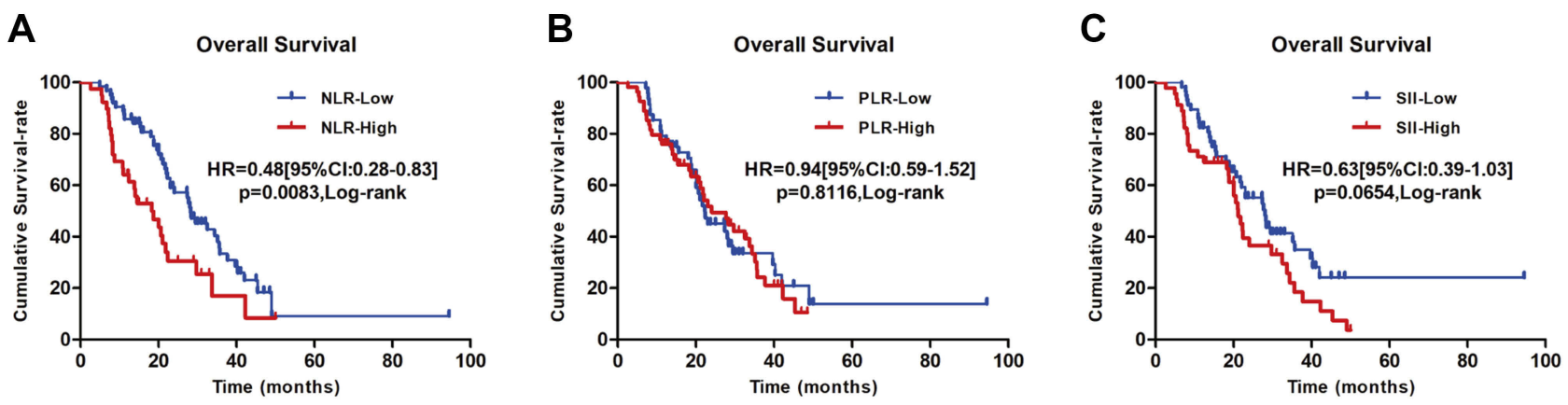

Figure 5 Kaplan-Meier curves of overall survival (OS) of mCRC patients according to baseline NLR (A), PLR (B), and SII (C).

Abbreviations: NLR, neutrophil-to-lymphocyte ratio; PLR, platelet-to-lymphocyte ratio; SIl, systemic immune-inflammation index; HR, hazard ratio; mCRC, metastatic colorectal cancer.

$(p=0.005)$ were significantly associated with OS (Figure 6A). In multivariate analysis, primary tumor resection (HR: 0.488 , 95\% CI: $0.302-0.788, p=0.003$ ) and early treatment response (HR: 0.393 , 95\% CI: $0.252-0.613, p<0.001$ ) were independent prognostic factors of OS (Figure 6B).

\section{Predictive value of inflammatory biomarkers as an indicator of early treatment response}

Early treatment response (response vs non-response) was associated with PFS and OS regarding inflammatory biomarkers (including NLR, PLR, and SII) (Table 2). In NLR $^{\text {Low }}$ group, PFS was significantly improved for patients with early treatment response compared to nonresponse (13.5 ms vs $7.8 \mathrm{~ms}$, HR: $1.61,95 \%$ CI: 1.07 $2.15, p=0.0032)$. However, early treatment response was not associated with OS $(p=0.1180)$. For NLR ${ }^{\text {High }}$ patients, early treatment response was not associated with PFS and OS ( $p=0.054$ and $p=0.051$, respectively).

Both in PLR ${ }^{\text {Low }}$ and PLR ${ }^{\text {High }}$ patients, PFS and OS were prolonged in early responders than non-responders $\left(\mathrm{PLR}^{\mathrm{Low}}, p=0.0074\right.$ and $p=0.0185$, respectively; $\mathrm{PLR}^{\mathrm{High}}$, $p=0.009$ and $p=0.0072$, respectively). Similar results are obtained in SII groups (SII ${ }^{\mathrm{Low}}, p=0.03$ and $p=0.037$, respectively; $\mathrm{SII}^{\mathrm{High}}, p=0.0070$ and $p=0.0411$, respectively).

\section{Discussion}

Substantial evidence showed that stroma-tumor interaction which led to a chronic inflammatory state was involved in carcinogenesis and tumor progression. ${ }^{24,25}$ Peripheral inflammatory cells including neutrophils, lymphocytes, and platelets were prognostic and predictive factors in various cancers like CRC. ${ }^{20,22,26-28}$ Neutrophils promoted adhesion and seeding of distant organ sites through secretion of circulating growth factors such as VEGF and proteases. ${ }^{29,30}$ Platelets induced circulating tumor cells epithelial-mesenchymal transition and promoted its extravasation to metastatic sites. ${ }^{31}$ On the contrary, lymphocytes played a crucial role in tumor defense by inducing cytotoxic cell death and inhibiting tumor cell proliferation and migration, thereby dictating the host's immune defense to malignancy. ${ }^{32}$ Thus, tumor inflammatory microenvironment modulation could influence cancer progression. Furthermore, tumor inflammatory microenvironment supported tumor progression and induced chemoresistance. ${ }^{15,24}$

In order to investigate the potential impact of surrogate markers of inflammatory reaction such as NLR, PLR, and, SII in mCRC treated with cetuximab, we showed that patients with low NLR were associated with better PFS and OS than those with high NLR. Meanwhile, elevated SII was significantly associated with poor PFS but not with OS. What is worth to mention that PLR was not significantly associated with either PFS or OS. In univariate analysis, in addition to the traditional prognostic factors (age, primary tumor resection, primary tumor location, CEA, and CT regime), NLR and SII were significantly associated with PFS and OS. In multivariate analyses, only NLR remained a prognostic factor for PFS. To date, our results are somewhat different from previous reports. Jing Yang et al, ${ }^{22}$ indicated that NLR was an independent prognostic factor not only for PFS but also for OS. Moreover, elevated PLR was significantly associated with poor PFS but not with OS, and SII was not significantly associated with PFS and OS. The major causes of these contrasting findings can be considered as follows: Firstly, its single-center study with selection bias. Secondly, the role of PLR in the 

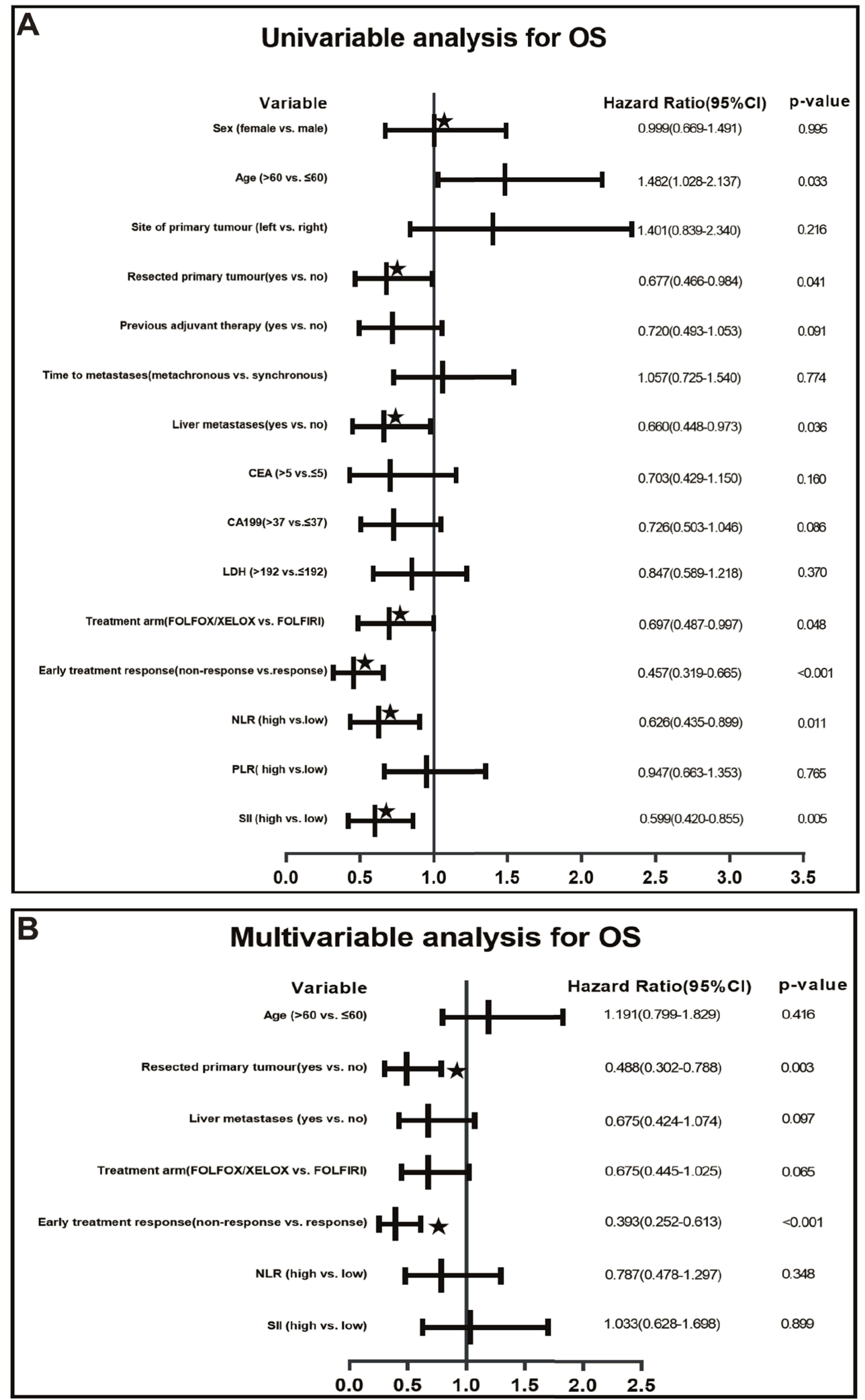

Figure 6 Forest plot illustrating the results of univariable $(\mathbf{A})$ and multivariable $(\mathbf{B})$ analysis of covariates associated with the overall survival in $\mathrm{mCRC} \star \star$ means $P<0.05$. Abbreviations: OA, overall survival; CEA, carcino-embryonic antigen; CAI99: carbohydrate antigen 19-9, LDH, lactate dehydrogenase; NLR, neutrophil-to-lymphocyte ratio; PLR, platelet-to-lymphocyte ratio; SII, systemic immune-inflammation index. 
Table 2 Predictive value of the inflammatory biomarkers as a function of early treatment response

\begin{tabular}{|c|c|c|c|c|c|c|c|}
\hline & \multirow{2}{*}{$\begin{array}{l}\text { Number of } \\
\text { event }\end{array}$} & \multicolumn{3}{|l|}{ PFS } & \multicolumn{3}{|l|}{ os } \\
\hline & & $\begin{array}{l}\text { Median of PFS, } \\
\text { months }(95 \% \mathrm{CI})\end{array}$ & $\begin{array}{l}\text { HR } \\
(95 \% \mathrm{Cl})\end{array}$ & $p$-value & $\begin{array}{l}\text { Median of PFS, } \\
\text { months }(95 \% \mathrm{CI})\end{array}$ & $\begin{array}{l}\text { HR } \\
(95 \% \mathrm{Cl})\end{array}$ & $p$-value \\
\hline \multicolumn{8}{|l|}{$N L R<3.285$} \\
\hline Response & 43 & $13.5(10.6-17.9)$ & 1.00 & 0.0032 & $29.5(24.0-32.9)$ & 1.00 & 0.1880 \\
\hline Non-response & 20 & $8.4(5.2-11.0)$ & $1.61(1.07-2.15)$ & & $23.2(I 7.5-28.8)$ & I.08 (0.54-I.62) & \\
\hline \multicolumn{8}{|l|}{ NLR $\geq 3.285$} \\
\hline Response & 10 & $12.9(10.5-16.3)$ & 1.00 & 0.0540 & $23.3(|6.8-3| .4)$ & 1.00 & 0.0510 \\
\hline Non-response & 29 & $7.8(5.3-10.8)$ & $2.18(1.90-2.59)$ & & I5.5 (II.8-19.2) & $1.62(1.22-2.03)$ & \\
\hline \multicolumn{8}{|l|}{ PLR < $17 \mid .45$} \\
\hline Response & 30 & 13.5 (6.5-19.9) & 1.00 & 0.0074 & $28.1(22.2-33.4)$ & 1.00 & 0.0185 \\
\hline Non-response & 18 & $8.4(5.9-11.7)$ & $1.61(1.10-2.11)$ & & I8.5 (I4.3-22.7) & $1.80(\mid .29-2.3 \mathrm{I})$ & \\
\hline \multicolumn{8}{|l|}{ PLR $\geq|7| .45$} \\
\hline Response & 23 & $13.4(10.3-16.9)$ & 1.00 & 0.0090 & $27.4(22.6-32.5)$ & 1.00 & 0.0072 \\
\hline Non-response & 31 & $6.0(4.4-9.8)$ & $2.23(1.72-2.74)$ & & $15.6(12.9-22.5)$ & $1.74(1.23-2.45)$ & \\
\hline \multicolumn{8}{|l|}{ SII <660.55 } \\
\hline Response & 36 & $12.8(7.3-19.1)$ & 1.00 & 0.0300 & $28.3(23.0-34.3)$ & 1.00 & 0.0320 \\
\hline Non-response & 21 & $8.9(6.4-12.1)$ & $1.44(0.93-1.95)$ & & $19.6(15.6-24.4)$ & I.5। (0.99-2.02) & \\
\hline \multicolumn{8}{|l|}{ SII $\geq 660.55$} \\
\hline Response & 17 & |3.5 (11.3-|5.8) & 1.00 & 0.0070 & $24.0(19.8-29.9)$ & 1.00 & $0.04 I I$ \\
\hline Non-response & 28 & $7.7(5.2-10.7)$ & $2.37(1.99-2.87)$ & & $15.4(|2.3-2| .8)$ & $1.56(1.07-2.05)$ & \\
\hline
\end{tabular}

Notes: $P$-value $<0.05$ was considered statistically significant.

Abbreviations: PFS, progression-free survival; OS, overall survival; $\mathrm{Cl}$, confidence interval; HR, hazard ratio; nLR, Neutrophil-to-lymphocyte ratio; PLR, platelet-tolymphocyte ratio; SII, systemic immune-inflammation index.

prognosis of CRC patients is still controversial. Several studies supported that pre-treatment PLR as a favorable marker for CRC patients while several studies with contrast conclusion. ${ }^{12,13,33-38}$ Thirdly, SII was only recently investigated as a prognostic factor in several types of tumors, and its prognostic value in CRC patients had been far from well defined. ${ }^{39-41}$ For those reasons, further studies should be performed to investigate the prognostic value of PLR and SII for the efficacy of cetuximab in mCRC patients.

The mechanism underlying the association between the chronic inflammation and malignant tumor is complex, but it could be due to the association of NLR with inflammation. Increasing evidence suggests that neutrophilia can inhibit the immune system, abolishing the cytolytic activity of immune cells. ${ }^{42,43}$ At the same time, both tumor cells and host cell, including neutrophils, can produce chemokines and cytokines, thus contributing to tumor progression. ${ }^{7}$ On the other hand, lymphocytic response is the main component of controlling cancer progression by inducing cytotoxic cell death and inhibiting tumor cell proliferation and migration, thereby dictating the host's immune response to malignancy. Additionally, neutrophilia suppresses lymphocyte activity by releasing reactive oxygen species, nitric oxide, and arginase, therefore hindering the antitumor immune response. ${ }^{44}$ In this way, high NLR which indicates high neutrophils counts and low lymphocyte counts are related to adverse prognosis in various solid tumors, including CRC. ${ }^{9-11,13}$ This is consistent with our results. Meanwhile, elevated SII indicates high neutrophils, high platelets and low lymphocytes which reflects both progression of cancer and weak immune status of the host. In our study, results of multivariate analysis suggested a tendency of improved OS in patients with high SII which showed no statistical significance. Therefore, further studies are expected to confirm the prognostic value of SII. However, it should be noted that whether neutrophils, lymphocytes or platelets, they 
are non-specific parameters because they are susceptible to comorbid diseases such as inflammation or infection. ${ }^{45}$ In our study, we specifically excluded patients with infectious diseases from the study in our exclusion criteria.

In the present study, the associations between inflammatory markers, the early treatment response, and clinic-pathological parameters, in addition, the outcome of patients with mCRC was retrospectively investigated. Our data suggested that in NLR, PLR, and SII low groups, more patients achieved early response than high groups. This study also confirmed that early treatment response was significantly associated with PFS and OS in univariate and multivariate analyses. Additionally, PFS and OS according to early response were also analyzed in different inflammatory marker groups. These results revealed that the significant differences on PFS and OS were universal in PLR and SII, despite the differences between the low and high groups. However, PFS showed that a significant difference was only existed in the low NLR group, but not in the high NLR group; meanwhile, there was no difference regarding OS. This may be due to the insufficient sample size (only 10 patients) in high NLR group with early response.

The present study has a number of limitations, including its retrospective nature, which may lead to bias in the data analysis, and the relatively small sample of patients received cetuximab. Thus, prospective, multi-center, and larger population studies are needed to validate these results.

In summary, our results showed a positive correlation between per-treatment inflammatory biomarkers (especially NLR) and PFS and OS in patients with $\mathrm{mCRC}$ treated with cetuximab in first line. Furthermore, per-treatment inflammatory biomarkers were indicators of early treatment response. This study provides a highly reproducible, easily obtainable, inexpensive, reliable, and practical index for predicting cetuximab efficacy, and to facilitate the administration of therapy in patients with a low NLR and early treatment response in order to achieve an improved response which would enhance the long-term outcomes for patients with mCRC. However, the potential underlying mechanisms and the performance of those inflammatory biomarkers in clinical practice should be validated in further prospective studies.

\section{Acknowledgment}

The study was supported by National Science Foundation of China (81502013 and 81672327) and SCORE Foundation (Y-MX2015-078) and Program of Shanghai Academic/Technology Research Leader (17XD1402600) and Program for Outstanding Medical Academic Leader and Shanghai Municipal Education CommissionGaofeng Clinical Medicine Grant Support (20161410) and Development Grant for Clinical Trial (SHDC12017X06).

\section{Disclosure}

The authors report no conflicts of interest in this work.

\section{References}

1. Van Cutsem E, Kohne CH, Lang I, et al. Cetuximab plus irinotecan, fluorouracil, and leucovorin as first-line treatment for metastatic colorectal cancer: updated analysis of overall survival according to tumor KRAS and BRAF mutation status. J Clin Oncol. 2011;29:2011-2019. doi:10.1200/JCO.2010.33.5091

2. Stintzing S, Modest DP, Rossius L, et al. FOLFIRI plus cetuximab versus FOLFIRI plus bevacizumab for metastatic colorectal cancer (FIRE-3): a post-hoc analysis of tumour dynamics in the final RAS wild-type subgroup of this randomised open-label phase 3 trial. Lancet Oncol. 2016;17:1426-1434. doi:10.1016/S1470-2045(16)30269-8

3. Venook AP, Niedzwiecki D, Lenz HJ, et al. Effect of first-line chemotherapy combined with cetuximab or bevacizumab on overall survival in patients with KRAS wild-type advanced or metastatic colorectal cancer: a randomized clinical trial. JAMA. 2017;317:2392-2401. doi:10.1001/jama.2017.7105

4. Qin S, Li J, Wang L, et al. Efficacy and tolerability of first-line cetuximab plus leucovorin, fluorouracil, and oxaliplatin (FOLFOX4) versus FOLFOX-4 in patients with RAS wild-type metastatic colorectal cancer: the open-label, randomized, Phase III TAILOR trial. J Clin Oncol. 2018;36(30):3031-3039. doi:10.1200/JCO.2018. 78.3183 .

5. Oda K, Matsuoka Y, Funahashi A, Kitano H. A comprehensive pathway map of epidermal growth factor receptor signaling. Mol Syst Biol. 2005;1:2005 0010. doi:10.1038/msb4100014

6. Yonesaka K, Zejnullahu K, Okamoto I, et al. Activation of ERBB2 signaling causes resistance to the EGFR-directed therapeutic antibody cetuximab. Sci Transl Med. 2011;3:99ra86. doi:10.1126/ scitranslmed.3002442

7. Balkwill F, Mantovani A. Cancer and inflammation: implications for pharmacology and therapeutics. Clin Pharmacol Ther. 2010;87:401406. doi:10.1038/clpt.2009.312

8. Diakos CI, Charles KA, McMillan DC, Clarke SJ. Cancer-related inflammation and treatment effectiveness. Lancet Oncol. 2014;15: e493-e503. doi:10.1016/S1470-2045(14)70263-3

9. Chua W, Charles KA, Baracos VE, Clarke SJ, Baracos VE, Clarke SJ. Neutrophil/lymphocyte ratio predicts chemotherapy outcomes in patients with advanced colorectal cancer. $B r \quad J$ Cancer. 2011;104:1288-1295. doi:10.1038/bjc. 2011.100

10. Absenger G, Szkandera J, Pichler M, et al. A derived neutrophil to lymphocyte ratio predicts clinical outcome in stage II and III colon cancer patients. Br J Cancer. 2013;109:395-400. doi:10.1038/bjc.2013.346

11. Clarke S, Burge M, Cordwell C, Gibbs P, Reece W, Tebbutt N. An Australian translational study to evaluate the prognostic role of inflammatory markers in patients with metastatic ColorEctal caNcer Treated with bevacizumab (Avastin) [ASCENT]. BMC Cancer. 2013;13:120. doi:10.1186/1471-2407-13-120

12. Li Y, Jia H, Yu W, et al. Nomograms for predicting prognostic value of inflammatory biomarkers in colorectal cancer patients after radical resection. Int J Cancer. 2016;139:220-231. doi:10.1002/ijc.30071

13. Wu Y, Li C, Zhao J, et al. Neutrophil-to-lymphocyte and platelet-tolymphocyte ratios predict chemotherapy outcomes and prognosis in patients with colorectal cancer and synchronous liver metastasis. World J Surg Oncol. 2016;14:289. doi:10.1186/s12957-016-1044-9 
14. Kim IH, Lee JE, Yang JH, Jeong JW, Ro S, Lee MA. Clinical significance of changes in systemic inflammatory markers and carcinoembryonic antigen levels in predicting metastatic colorectal cancer prognosis and chemotherapy response. Asia Pac J Clin Oncol. 2018;14:239-246. doi:10.1111/ajco.12784

15. de Visser KE, Jonkers J. Towards understanding the role of cancerassociated inflammation in chemoresistance. Curr Pharm Des. 2009;15:1844-1853. doi:10.2174/138161209788453239

16. Jinushi M, Komohara Y. Tumor-associated macrophages as an emerging target against tumors: creating a new path from bench to bedside. Biochim Biophys Acta. 2015;1855:123-130. doi:10.1016/j.bbcan.2015. 01.002

17. Olive KP. Fanning the flames of cancer chemoresistance: inflammation and anticancer therapy. J Oncol Pract. 2017;13:181-183. doi:10.1200/JOP.2017.021154

18. Sanderson RD, Elkin M, Rapraeger AC, Ilan N, Vlodavsky I. Heparanase regulation of cancer, autophagy and inflammation: new mechanisms and targets for therapy. FEBS J. 2017;284:42-55. doi:10.1111/febs. 13932

19. Van Glabbeke M, Verweij J, Casali PG, et al. Initial and late resistance to imatinib in advanced gastrointestinal stromal tumors are predicted by different prognostic factors: a European Organisation for Research and Treatment of Cancer-Italian Sarcoma GroupAustralasian Gastrointestinal Trials Group study. J Clin Oncol. 2005;23:5795-5804. doi:10.1200/JCO.2005.11.601

20. Sun $\mathrm{H}, \mathrm{Hu} \mathrm{P}, \mathrm{Du} J$, Wang $X$. Predictive value of inflammatory indexes on the chemotherapeutic response in patients with unresectable lung cancer: a retrospective study. Oncol Lett. 2018;15:40174025. doi:10.3892/ol.2018.7781

21. Dell'Aquila E, Cremolini C, Zeppola T, et al. Prognostic and predictive role of neutrophil/lymphocytes ratio in metastatic colorectal cancer: a retrospective analysis of the TRIBE study by GONO. Ann Oncol. 2018;29:924-930. doi:10.1093/annonc/mdy004

22. Yang J, Guo X, Wang M, Ma X, Ye X, Lin P. Pre-treatment inflammatory indexes as predictors of survival and cetuximab efficacy in metastatic colorectal cancer patients with wild-type RAS. Sci Rep. 2017;7:17166. doi:10.1038/s41598-017-17130-6

23. Watanabe H, Okada M, Kaji Y, et al. [New response evaluation criteria in solid tumours-revised RECIST guideline (version 1.1)] Gan To Kagaku Ryoho. 2009;36:2495-2501.

24. Coussens LM, Werb Z. Inflammation and cancer. Nature. 2002;420:860-867. doi:10.1038/nature01322

25. Roxburgh CS, McMillan DC. Role of systemic inflammatory response in predicting survival in patients with primary operable cancer. Future Oncol. 2010;6:149-163. doi:10.2217/fon.09.136

26. Goto W, Kashiwagi S, Asano Y, et al. Predictive value of lymphocyte-to-monocyte ratio in the preoperative setting for progression of patients with breast cancer. BMC Cancer. 2018;18:1137. doi:10.1186/ s12885-018-4242-8

27. Yang $\mathrm{J}, \mathrm{Xu} \mathrm{H}$, Guo X, et al. Pretreatment inflammatory indexes as prognostic predictors for survival in colorectal cancer patients receiving neoadjuvant chemoradiotherapy. Sci Rep. 2018;8:3044. doi:10.1038/s41598-018-21093-7

28. Deng Q, He B, Liu X, et al. Prognostic value of pre-operative inflammatory response biomarkers in gastric cancer patients and the construction of a predictive model. J Transl Med. 2015;13:66. doi:10.1186/s12967-015-0541-x

29. Cools-Lartigue J, Spicer J, McDonald B, et al. Neutrophil extracellular traps sequester circulating tumor cells and promote metastasis. $J$ Clin Invest. 2013. doi:10.1172/JCI67484
30. Rossi L, Santoni M, Crabb SJ, et al. High neutrophil-to-lymphocyte ratio persistent during first-line chemotherapy predicts poor clinical outcome in patients with advanced urothelial cancer. Ann Surg Oncol. 2015;22:1377-1384. doi:10.1245/s10434-014-4097-4

31. Labelle M, Begum S, Hynes RO. Direct signaling between platelets and cancer cells induces an epithelial-mesenchymal-like transition and promotes metastasis. Cancer Cell. 2011;20:576-590. doi:10.1016/j. ccr.2011.09.009

32. De Giorgi U, Mego M, Scarpi E, et al. Relationship between lymphocytopenia and circulating tumor cells as prognostic factors for overall survival in metastatic breast cancer. Clin Breast Cancer. 2012;12:264-269. doi:10.1016/j.clbc.2012.04.004

33. Szkandera J, Pichler M, Absenger G, et al. The elevated preoperative platelet to lymphocyte ratio predicts decreased time to recurrence in colon cancer patients. Am J Surg. 2014;208:210-214. doi:10.1016/j. amjsurg.2013.10.030

34. Passardi A, Scarpi E, Cavanna L, et al. Inflammatory indexes as predictors of prognosis and bevacizumab efficacy in patients with metastatic colorectal cancer. Oncotarget. 2016;7:33210-33219. doi:10.18632/oncotarget.8901

35. Azab B, Shah N, Radbel J, et al. Pretreatment neutrophil/lymphocyte ratio is superior to platelet/lymphocyte ratio as a predictor of longterm mortality in breast cancer patients. Med Oncol. 2013;30:432. doi:10.1007/s12032-012-0432-4

36. Zou ZY, Liu HL, Ning N, Li SY, Du XH, Li R. Clinical significance of pre-operative neutrophil lymphocyte ratio and platelet lymphocyte ratio as prognostic factors for patients with colorectal cancer. Oncol Lett. 2016;11:2241-2248. doi:10.3892/ol.2016.4216

37. Ozawa T, Ishihara S, Kawai K, et al. Impact of a lymphocyte to monocyte ratio in stage IV colorectal cancer. J Surg Res. 2015;199:386-392. doi:10.1016/j.jss.2015.06.014

38. Ying HQ, Deng QW, He BS, et al. The prognostic value of preoperative NLR, d-NLR, PLR and LMR for predicting clinical outcome in surgical colorectal cancer patients. Med Oncol. 2014;31:305. doi:10.1007/s12032-014-0374-0

39. Feng JF, Chen S, Yang X. Systemic immune-inflammation index (SII) is a useful prognostic indicator for patients with squamous cell carcinoma of the esophagus. Medicine. 2017;96:e5886. doi:10.1097/MD.0000 000000005886

40. Hong X, Cui B, Wang M, Yang Z, Wang L, Xu Q. Systemic immuneinflammation index, based on platelet counts and neutrophil-lymphocyte ratio, is useful for predicting prognosis in small cell lung cancer. Tohoku J Exp Med. 2015;236:297-304. doi:10.1620/tjem.236.297

41. Huang L, Liu S, Lei Y, et al. Systemic immune-inflammation index, thymidine phosphorylase and survival of localized gastric cancer patients after curative resection. Oncotarget. 2016;7:44185-44193. doi:10.18632/oncotarget.9923

42. Petrie HT, Klassen LW, Kay HD. Inhibition of human cytotoxic T lymphocyte activity in vitro by autologous peripheral blood granulocytes. J Immunol. 1985;134(1):230-234.

43. el-Hag A, Clark RA. Immunosuppression by activated human neutrophils. Dependence on the myeloperoxidase system. J Immunol. 1987;139(7):2406-2413

44. Lee S, Oh SY, Kim SH, et al. Prognostic significance of neutrophil lymphocyte ratio and platelet lymphocyte ratio in advanced gastric cancer patients treated with FOLFOX chemotherapy. BMC Cancer. 2013;13:350. doi:10.1186/1471-2407-13-350

45. Azab B, Jaglall N, Atallah JP, et al. Neutrophil-lymphocyte ratio as a predictor of adverse outcomes of acute pancreatitis. Pancreatology. 2011;11(4):445-452. doi:10.1159/000331494 


\section{Publish your work in this journal}

Cancer Management and Research is an international, peer-reviewed open access journal focusing on cancer research and the optimal use of preventative and integrated treatment interventions to achieve improved outcomes, enhanced survival and quality of life for the cancer patient.
The manuscript management system is completely online and includes a very quick and fair peer-review system, which is all easy to use. Visit http://www.dovepress.com/testimonials.php to read real quotes from published authors.

Submit your manuscript here: https://www.dovepress.com/cancer-management-and-research-journal 